\title{
Functional Analysis of the Helicobacter pylori cag Pathogenicity Island Reveals Both VirD4-CagA-Dependent and VirD4-CagA-Independent Mechanisms
}

\author{
Matthias Selbach, Stefan Moese, Thomas F. Meyer,* and Steffen Backert \\ Abteilung Molekulare Biologie, Max-Planck-Institut für Infektionsbiologie, D-10117 Berlin, Germany
}

Received 13 August 2001/Returned for modification 16 October 2001/Accepted 8 November 2001

\begin{abstract}
The type IV secretion machinery encoded by the cag pathogenicity island (PAI) of Helicobacter pylori has been implicated in a series of host responses during infection. Here, we analyzed the function of 12 cag PAI genes from both $c a g$ I and $c a g$ II loci, including the complete $\operatorname{virB} / D$ complex (virB4, virB7, virB8, virB9, virB10, virB11, and virD4). We monitored interleukin-8 (IL-8) secretion, CagA translocation and tyrosine phosphorylation, and induction of a scattering ("hummingbird") phenotype upon $\mathrm{H}$. pylori infection of AGS gastric epithelial cells. For the first time, we have complemented individual cag PAI gene knockout mutants with their intact genes expressed from a shuttle vector and showed that complemented CagA and VirD4 restored wild-type function. Our results demonstrate that phenotypic changes and phosphorylation of CagA depended on all virB/D genes and several other genes of the cag PAI. Induction of IL-8 secretion depended largely on the same set of genes but was independent of CagA and VirD4. Thus, CagA translocation and induction of IL-8 secretion are regulated by VirD4-CagA-dependent and VirD4-CagA-independent mechanisms, respectively. The function of VirD4 as a possible adapter protein which guides CagA into the type IV secretion channel is presented in a model.
\end{abstract}

The human pathogen Helicobacter pylori is a gram-negative bacterium that resides in the stomach of half of the world's population. H. pylori has been identified as the causative agent of chronic inflammation, chronic gastritis, and peptic ulceration and was considered a risk factor for the development of mucosa-associated lymphoid tissue lymphoma and adenocarcinoma of the stomach $(15,27)$. The active phase of $H$. pyloriinduced gastritis is characterized by a dense infiltration of neutrophils into the lamina propria that is believed to contribute substantially to tissue damage.

Several in vitro and in vivo studies have demonstrated that $H$. pylori induces host cell vacuolation $(17,33)$, cytoskeletal rearrangements $(5,11,36)$, and the secretion of a range of inflammatory mediators, including cytokines interleukin (IL)-1, IL-6, and tumor necrosis factor alpha and chemokines IL-8, Gro- $\alpha$, RANTES, ENA-78, MCP-1, and Mip- $1 \alpha$ (8, 9, 25, 41). Of these factors, IL- 8 is a potent neutrophil and T-cell chemoattractant and activator, which is believed to play a key role in the pathogenesis of $H$. pylori-induced tissue damage (31, $34,42) . H$. pylori is thought to stimulate IL- 8 gene expression by activating the transcription factor nuclear factor $\kappa \mathrm{B}(19,37)$.

Several $H$. pylori virulence factors have been determined. First, bacterial adhesion is essential for host-pathogen interaction. The Lewis b-binding adhesin BabA (21) and two outermembrane proteins, designated AlpA and AlpB (30), have been identified. Second, patients with gastritis, ulcers, or malignancies are often infected with $H$. pylori type I strains. Although type I and type II strains do not constitute two distinct

\footnotetext{
* Corresponding author. Mailing address: Max-Planck-Institut für Infektionsbiologie, Abt. Molekulare Biologie, Schumannstr. 20/21, D-10117 Berlin, Germany. Phone: 493028460 400. Fax: 49302846 04 01. E-mail: meyer@mpiib-berlin.mpg.de.
}

lineages, some virulence factors have been reported to be prevalent in type I strains (14). These strains frequently express the adhesin BabA, cytotoxic alleles of the vacuolating cytotoxin (VacA), and the immunodominant cytotoxin-associated antigen (CagA). While VacA induces the formation of vacuoles in infected cells (33), CagA has been associated with rearrangements of the actin cytoskeleton resulting in phenotypic changes called the scattering or "hummingbird" phenotype $(5,36)$.

Genetically, type I strains are characterized by the presence of a 40-kb DNA segment (containing up to 31 open reading frames [ORFs]) termed the cag pathogenicity island (PAI) that is often divided into the cag I and cag II loci $(9,14)$. While CagA is encoded within the cag PAI, VacA is encoded outside the pathogenicity island. Some of the cag PAI ORFs show significant homology to the virulence (vir) genes virB4, virB7, virB8, virB9, virB10, virB11, and virD4 of the so-called VirB/D complex of type IV secretion systems known from Agrobacterium tumefaciens and Bordetella pertussis $(10,14)$. Type IV systems can be regarded as molecular syringes that inject their specific substrate into the cytosol of target cells. They are involved in conjugative DNA transfer of prokaryotes and in the delivery of bacterial virulence factors into the cells of their eukaryotic hosts.

Recent studies have revealed compelling evidence that the cag PAI of $H$. pylori encodes a functional type IV machinery that translocates the CagA protein into the cytosol of gastric epithelial cells $(3,5,7,29,36,38,43)$. Translocated CagA is tyrosine-phosphorylated by an unidentified host kinase and undergoes processing into $\mathrm{p} 100^{\mathrm{CagA}}$ and $\mathrm{p} 35^{\mathrm{CagA}}$ fragments that have been observed in both gastric epithelial cells (6) and phagocytic cells $(26,28)$. However, their importance is not understood. Conspicuously, the IL-8-inducing activity of some H. pylori strains mutated in a series of cag PAI genes was 
strongly reduced $(1,9,23,40)$. These data led to the speculation that $H$. pylori induces IL- 8 secretion by an unidentified factor that is translocated through the type IV secretion system. However, there are also conflicting reports in which inactivation of virD4, virB10, and virB11 enhanced IL-8 secretion $(16,18)$. Another group has reported that water-soluble extracts of $H$. pylori containing nonprotein low-molecular-weight compounds can induce IL-8 secretion (22). These phenomena are not understood.

Thus, there are still open questions concerning the function of the type IV secretion system. A systematic functional analysis using genes throughout the cag I and cag II loci and different read-out systems (for the function of the type IV secretion system) in parallel has not yet been carried out. Moreover, in almost all recent studies, possible polar effects of cag PAI mutagenesis on expression of neighboring genes were not investigated. No studies using genetic complementation of individual cag PAI genes have been performed so far.

In this study, we have generated a large set of isogenic cag PAI mutants, including all virB $/ D$ genes, and analyzed their ability to induce IL- 8 secretion, CagA phosphorylation, and phenotypic changes. To rule out the possibility of polar effects in our mutants, we complemented two of our knockout strains with wild-type genes in trans. Our results demonstrate that CagA translocation and induction of IL-8 secretion depend on all vir homologs and other cag PAI genes. While phenotypic changes were found to be strictly correlated with CagA translocation, IL-8 release was independent of CagA. VirD4 played a special role, as it was essential for CagA translocation but dispensable for the induction of IL- 8 secretion, indicating important differences between these processes.

\section{MATERIALS AND METHODS}

H. pylori strains and production of cag PAI mutants. H. pylori strains P1, P12, G27, and J99 are clinical isolates and have been described previously (2, 9, 35). J99 is one of the sequenced strains and known to possess a continuous cag PAI. The cag PAI structure of strains P1, P12, and G27 is unknown.

We constructed isogenic cagA, $\operatorname{cagH}$, and vir gene knockout mutants in strain $\mathrm{P} 1$ by insertion of a chloramphenicol resistance gene cassette $\left(\mathrm{Cm}^{\mathrm{r}}, 1-\mathrm{kb}\right.$ Bam HI/ $B g l$ II fragment of plasmid pTnMax1) using a protocol described previously (7, 26). $\Delta c a g F, \Delta c a g I$, and $\Delta c a g M$ knockout mutants were created in strain G27 by transposon-mediated mutagenesis and were provided by Antonello Covacci (9). The mutant lacking the complete $c a g$ PAI $(\Delta \mathrm{PAI})$ was derived from strain P12 by insertion of a kanamycin resistance cassette as described previously (28). The $\mathrm{P} 12 \Delta v a c A$ and $\mathrm{P} 1 \Delta a l p A / B$ double mutants were kindly provided by Rainer Haas $(30,35)$.

H. pylori strains were cultivated on horse serum agar plates supplemented with vancomycin $(10 \mu \mathrm{g} / \mathrm{ml})$, nystatin $(1 \mu \mathrm{g} / \mathrm{ml})$, and trimethoprim $(5 \mu \mathrm{g} / \mathrm{ml})$. Depending on the specific strain, chloramphenicol (4 to $6 \mu \mathrm{g} / \mathrm{ml})$ and/or kanamycin $(20 \mu \mathrm{g} / \mathrm{ml})$ was added. Bacteria were cultivated for 2 days at $37^{\circ} \mathrm{C}$ in an anaerobic jar containing a Campygen gas mix of $5 \% \mathrm{O}_{2}, 10 \% \mathrm{CO}_{2}$, and $85 \% \mathrm{~N}_{2}$ (Oxoid, Wesel, Germany).

Complementation of CagA and VirD4. For construction of complementation vector constructs, cagA and virD4 were amplified from strain 26695 by PCR (NCBI database accession numbers AAD07614 and D64585, respectively). For $\operatorname{cag} A$, the primers 5'GTCGACCATCTTTAGCGTTGCATTTGATTT (forward) and 5'GTCGACCAACACAAGTAGCCCCTAAAACTT (reverse) were used. These primers contained unique $A c c$ I sites that were used to clone the PCR product into the ClaI site of the Escherichia coli/H. pylori shuttle vector $\mathrm{pHel} 2$ containing the oriT of RP4 and a kanamycin resistance gene cassette (aph-A3) as a selectable marker (20).

virD4 was amplified using the primers 5' TCCATATGGAAGACTTTTTGTA TAACAC (forward) and 5' CGGGATCCGACACACTTCTACTATCTTATG (reverse). The forward primer contained an $\mathrm{NdeI}$ site, and the reverse primer contained a Bam $\mathrm{HI}$ site for cloning in pHel2. virD4 was expressed under the constitutive $H$. pylori flaA promoter, which was cloned as described (20). The cagA and virD4 complementation constructs were transferred into $H$. pylori by conjugation of E. coli XL1-Blue with $\mathrm{P} 1 \Delta c a g A$ and $\mathrm{P} 1 \Delta v i r D 4$, respectively, using E. coli GC7[pRK2013] as the mobilizer (20). Plasmids were reisolated from transformed $H$. pylori strains, and restriction fragment length polymorphism patterns were confirmed in order to exclude integration of the constructs into the bacterial chromosome.

Synchronized infection assays. AGS cells (ATCC CRL 1739; a human gastric adenocarcinoma epithelial cell line) were cultivated in six-well tissue culture dishes in $2 \mathrm{ml}$ of RPMI 1640 medium (Gibco-BRL, Eggenstein, Germany) supplemented with $10 \%$ heat-inactivated fetal bovine serum (Gibco-BRL) per well for 2 days to reach monolayers of about $70 \%$ confluence. $H$. pylori cells were suspended in phosphate-buffered saline (PBS) and added to AGS cells at a multiplicity of infection (MOI) of 100 . In order to synchronize the infection, bacteria were centrifuged onto the cells for $5 \mathrm{~min}$ at $600 \times \mathrm{g}$. The alp $A / B$ mutant, which is defective for adherence, was added to the cells without centrifugation.

After incubation in a $5 \% \mathrm{CO}_{2}-95 \%$ air incubator for $5 \mathrm{~h}$, the phenotype was analyzed by phase contrast microscopy. When more than $60 \%$ of the cells were elongated (see Fig. 3), the hummingbird phenotype was considered to have been induced. After $6 \mathrm{~h}$, the cell culture medium was collected for the IL- 8 assay and stored at $-20^{\circ} \mathrm{C}$. Cells were washed once with ice-cold PBS containing $1 \mathrm{mM}$ $\mathrm{Na}_{3} \mathrm{VO}_{4}$ (Sigma-Aldrich, Deisenhofen, Germany), harvested with a rubber policeman in the same buffer, and pelleted together with attached bacteria at $600 \times$ $g$ and $4^{\circ} \mathrm{C}$

SDS-PAGE and immunoblotting. Cell pellets with attached bacteria were mixed with equal amounts of $2 \times$ sodium dodecyl sulfate-polyacrylamide gel electrophoresis (SDS-PAGE) buffer (100 mM Tris-HCl [pH 6.8], 5\% $\beta$-mercaptoethanol, $5 \%$ SDS, $0.2 \%$ bromophenol blue, $20 \%$ glycerol) and boiled for $5 \mathrm{~min}$. Proteins were separated by SDS-PAGE on $6 \%$ polyacrylamide gels and blotted onto polyvinylidene difluoride membranes (Immobilon-P; Millipore, Bedford, Mass.) using standard protocols. Membranes were blocked in TBS-T (140 mM $\mathrm{NaCl}, 25 \mathrm{mM}$ Tris- $\mathrm{HCl}$ [pH 7.4], $0.1 \%$ Tween 20 ) with $3 \%$ bovine serum albumin for $1 \mathrm{~h}$ at room temperature.

Tyrosine-phosphorylated proteins were detected with the monoclonal phosphotyrosine-specific antibody PY99 (Santa Cruz Biotechnology, Santa Cruz, Calif.), in a 1:1,000 dilution in TBS-T for $1 \mathrm{~h}$ at room temperature. CagA was detected with a polyclonal rabbit anti-CagA antibody, a gift of S. Censini and A. Covacci (12). Blots were washed three times in TBS-T and incubated with horseradish peroxidase-conjugated anti-mouse or anti-rabbit immunoglobulin secondary antibodies (Amersham Pharmacia Biotech) for $1 \mathrm{~h}$. After three more washing steps, the bound antibodies were detected with the Renaissance Western blot kit system for enhanced chemiluminescence immunostaining (ICN Biochemicals, Eschwege, Germany). The same blot was used for both antiphosphotyrosine and anti-CagA immunoblotting. Between the blotting procedures, the membranes were stripped for $30 \mathrm{~min}$ at $50^{\circ} \mathrm{C}$ in stripping buffer $(62.5 \mathrm{mM}$ Tris- $\mathrm{HCl}$ [pH 6.7], $100 \mathrm{mM}$ 2-mercaptoethanol, $2 \%$ SDS).

IL-8 ELISA. The amount of IL-8 secreted into the cell culture medium after $6 \mathrm{~h}$ of infection was determined by sandwich enzyme-linked immunosorbent assay (ELISA) using the CytoSets system (BioSource International, Camarillo, Calif.) according to the manufacturer's instructions. All samples were measured in triplicate from at least three independent experiments.

\section{RESULTS}

The H. pylori type IV secretion apparatus is thought to play an important role in host-pathogen interaction. In order to understand the role and function of this transporter system during infection, we used isogenic knockout mutants of 12 individual cag PAI genes in both the cag I and cag II loci, including all virB/D genes (Fig. 1). Three $H$. pylori isogenic mutants that lack either the complete cag PAI or vacA or $\operatorname{alp} A / B$ served as controls. In order to analyze the importance of individual $H$. pylori genes in the infection process, AGS gastric epithelial cells were infected with these $H$. pylori strains, and the host response was monitored using three different read-out systems: (i) IL-8 secretion, (ii) translocation and 


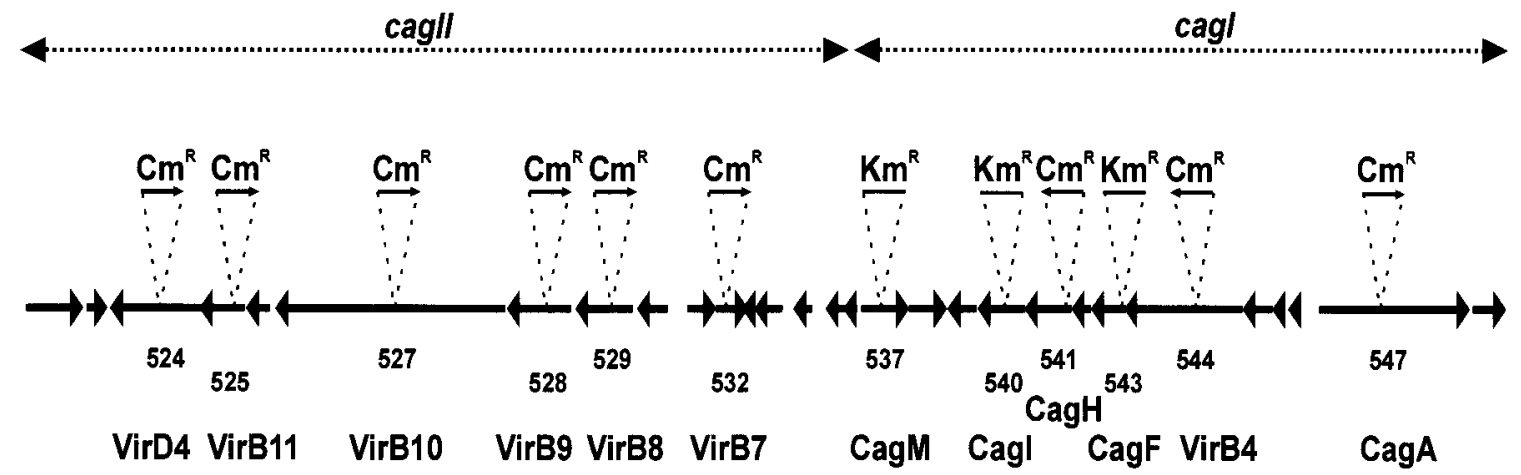

FIG. 1. Mutagenesis of the $H$. pylori cag PAI. In this schematic representation of the cag PAI, genes are numbered according to the nomenclature of TIGR strain 26695 (39). In some $H$. pylori isolates, the cag PAI can be divided into the cagI and cagII loci, as indicated (9). Twelve cag PAI genes that were inactivated by insertion of a chloramphenicol $\left(\mathrm{Cm}^{\mathrm{r}}\right)$ or a kanamycin $\left(\mathrm{Km}^{\mathrm{r}}\right)$ resistance cassette in the indicated position and direction are marked.

phosphorylation of CagA, and (iii) induction of a scattering (hummingbird) phenotype.

IL-8 levels in the cell culture supernatants were determined by ELISA (Fig. 2A). In parallel experiments, CagA translocation and tyrosine phosphorylation were analyzed by Western blotting using an antiphosphotyrosine antibody (Fig. 2B) and an anti-CagA antibody (Fig. 2C). Phenotypic changes in infected AGS cells were studied by phase contrast microscopy (Fig. 3). All data were summarized in Table 1.

Our results collectively demonstrated that translocation and phosphorylation of CagA, IL-8 secretion, and induction of the hummingbird phenotype were independent of VacA but depended strongly on (i) close contact between bacteria and host cells and (ii) the functional integrity of the type IV secretion system encoded in the cag PAI. Our studies also revealed distinct differences between these processes, namely, the distinction between a VirD4-CagA-independent host response (IL-8 secretion) and VirD4-CagA-dependent processes (translocation and tyrosine phosphorylation of CagA and induction of the hummingbird phenotype).

Infection of AGS cells with type I H. pylori wild-type strains P1, P12, and G27 induced IL-8 secretion (Fig. 2A) and translocation

A

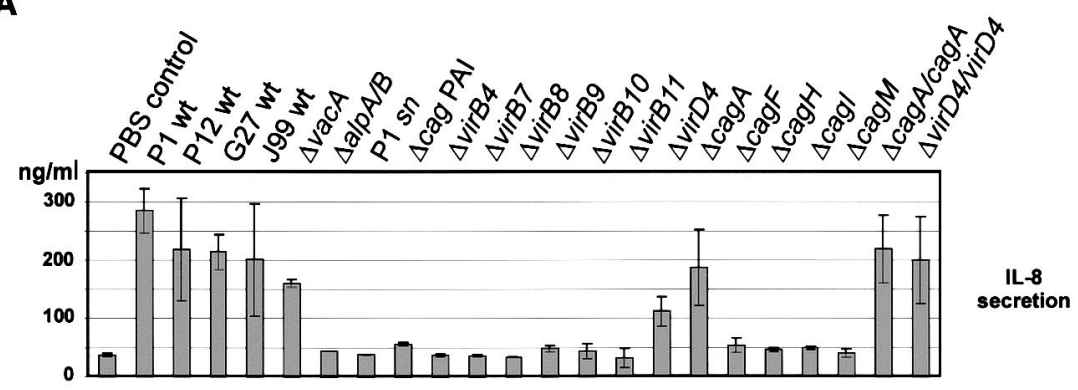

B

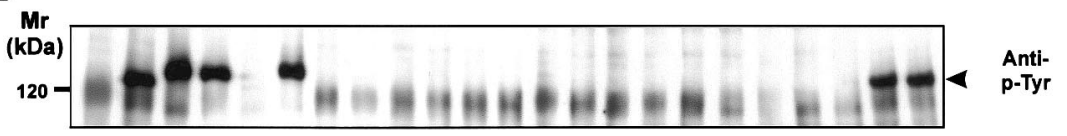

C

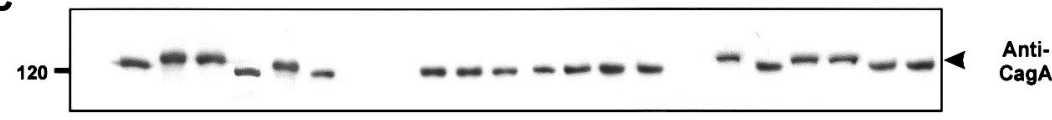

FIG. 2. Functional analysis of the $H$. pylori cag PAI. AGS cells were incubated with $H$. pylori wild-type (wt) strains, isogenic knockout mutants, or bacterial culture supernatant (sn) as indicated. (A) IL-8 secretion of AGS cells induced by H. pylori. Six hours after infection, the concentration of IL-8 secreted into the medium was determined by ELISA. While AGS cells infected with wild-type bacteria secrete large amounts of IL-8, none of the cag PAI gene knockout strains except the cagA and virD mutants induced IL-8 secretion above the control level. Error bars show standard deviation. (B) Tyrosine phosphorylation of translocated CagA (arrowhead) in AGS cells. Proteins from H. pylori-infected cells and attached bacteria were separated by SDS-PAGE, and tyrosine phosphorylation was investigated by immunoblotting with antiphosphotyrosine (anti-p-Tyr). Since no bacterial tyrosine kinase has been observed in $H$. pylori $(28,39)$, detection of tyrosine-phosphorylated CagA indicated successful translocation from the bacterium into host cells. All genes of the cag PAI analyzed in this study were essential for CagA translocation. Complementation of the cagA and virD4 knockout mutants in trans $(\Delta c a g A / \operatorname{cag} A$ and $\Delta v i r D 4 / v i r D 4$, respectively) restored CagA translocation. (C) Immunoblot analysis of CagA protein expression. The blot in B was stripped and reprobed with a CagA-specific antiserum. Inactivation of flanking cag PAI genes did not alter the expression of CagA (arrowhead). 

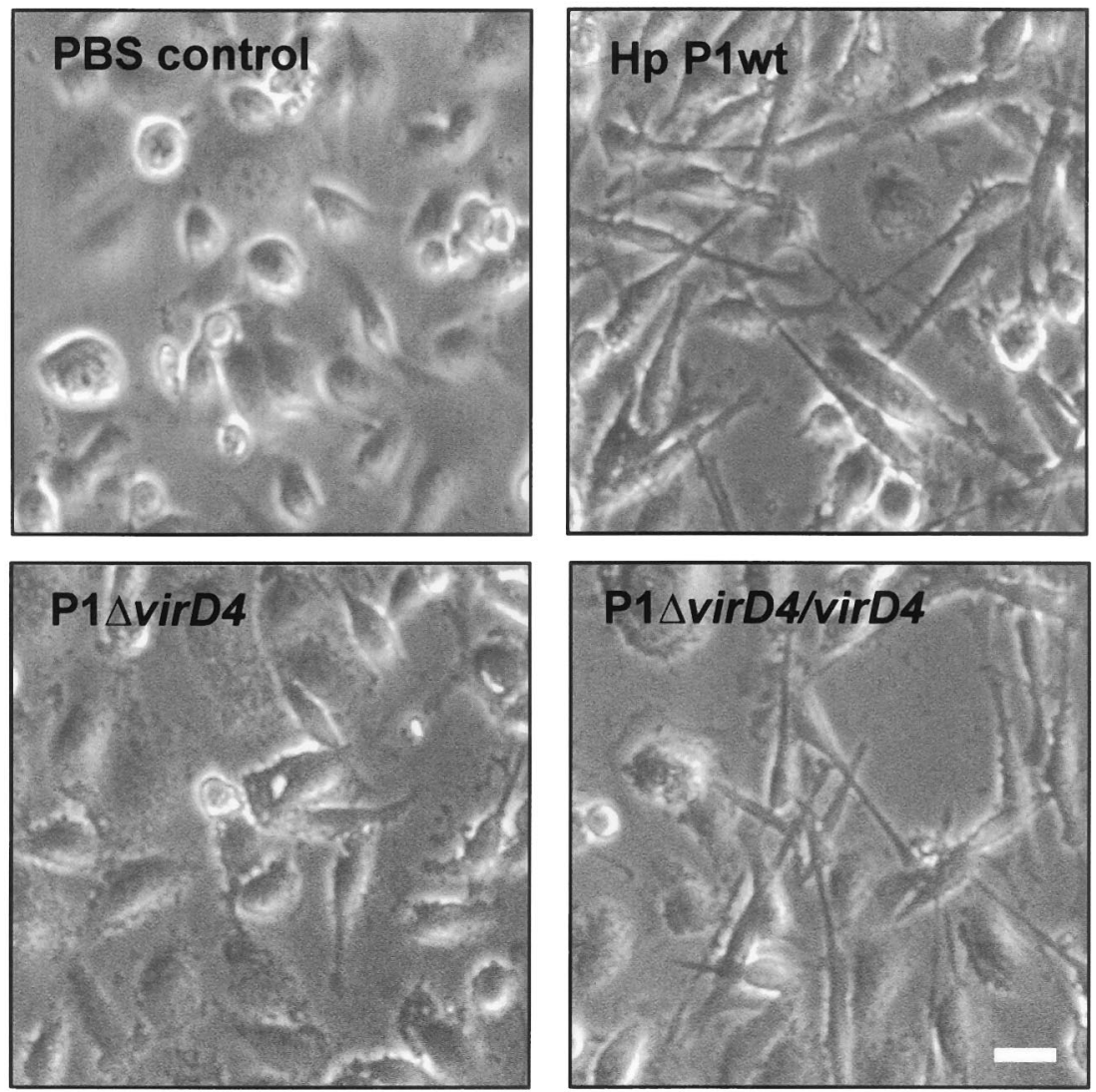

FIG. 3. Induction of the scattering (hummingbird) phenotype in AGS cells by H. pylori depends on functional vir and cagA genes. Phase contrast micrographs of AGS cells infected with wild-type strain P1 and an isogenic virD4 knockout mutant (P1 $\Delta$ virD4) are shown as an example. Infection with P1svirD4 complemented with intact VirD4 expressed from shuttle vector pHel2 (P1svirD4/virD4) restored the hummingbird phenotype. In each experiment, $H$. pylori infection was for $5 \mathrm{~h}$ at an MOI of 100 . The hummingbird phenotype was considered induced when more than $60 \%$ of the infected AGS cells were elongated, as shown in the right panels. Bar, $10 \mu \mathrm{m}$.

and phosphorylation of CagA (Fig. 2B and C). Cells infected with these wild-type strains were significantly elongated ( 25 to $70 \mu \mathrm{m})$ and had a spindle-like morphology in more than $60 \%$ of the infected cells (Fig. 3) that has been referred to as the scattering or hummingbird phenotype $(5,36)$. An exception was wild-type strain J99, which induced IL-8 secretion but no tyrosine phosphorylation of CagA or phenotypic changes in host cells. This might be due to the lack of tyrosine phosphorylation motifs $(5,29)$. An alp $A / B$ mutant failed to induce any of these host responses. None of the investigated host responses were induced by $H$. pylori culture supernatant (Fig. 2). This indicated that no bacterial factor secreted into the culture medium is responsible for IL-8 secretion, translocation and phosphorylation of CagA, and induction of the hummingbird phenotype.

In agreement with these results, we found that all virB gene knockout strains $(\Delta \operatorname{virB4}, \operatorname{virB7}, \operatorname{virB8}, \operatorname{virB9}, \operatorname{virB10}$, and virB11) of the contact-dependent type IV transporter complex and a mutant lacking the entire cag PAI were defective for IL-8 secretion (Fig. 2A), translocation and phosphorylation of CagA (Fig. 2B and C), and induction of the hummingbird phenotype (Fig. 3, Table 1), demonstrating their essential function in each of these host responses. Moreover, several other genes of the cag PAI (cagF, cagH, cagI, and cagM) having no significant homology to other known genes or gene products were also needed for these processes. Hence, the ability of the bacteria to stimulate IL-8 production largely correlated with their ability to actively translocate CagA (Table 1).

We observed two interesting exceptions. First, the $\operatorname{cag} A$ knockout strain stimulated a 5.1-fold increase in IL-8 release by infected AGS cells compared to uninfected controls. This IL-8 level is similar to levels obtained during infection with wild-type $H$. pylori, indicating that CagA as a translocated effector protein does not play a role in IL-8 induction. The second exception was the virD4 knockout strain. Although the intact virD4 gene was essential for translocation and phosphorylation of CagA (Fig. 2B and C) and induction of the hummingbird phenotype (Fig. 3), inactivation of the virD4 gene did not abolish IL-8 secretion compared to all other cag PAI mutant strains but reduced the IL-8 level by about $60 \%$ (Fig. 2A). As the IL-8 release stimulated by this mutant strain was significantly above background levels, virD4 can be considered nonessential for induction of IL-8 release.

In order to rule out polar effects of our insertional mutagenesis,

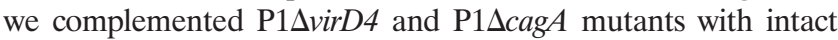
virD4 and cagA, respectively, expressed from the E. coli-H. pylori shuttle vector pHel2. The complemented strains, P1 $\Delta$ cagA/ cagA and P1AvirD4/virD4, respectively, induced transloca- 
TABLE 1. Translocation and tyrosine phosphorylation of CagA, induction of the scattering phenotype, and secretion of IL-8 in AGS cells infected with H. pylori ${ }^{a}$

\begin{tabular}{|c|c|c|c|c|c|}
\hline $\begin{array}{l}\text { Bacterial } \\
\text { strain }\end{array}$ & $\begin{array}{l}\text { Mutated } \\
\text { ORF }^{b}\end{array}$ & $\begin{array}{l}\text { CagA } \\
\text { expres- } \\
\text { sion }\end{array}$ & $\begin{array}{l}\text { CagA trans- } \\
\text { location/phos- } \\
\text { phorylation }\end{array}$ & $\begin{array}{c}\text { Induction of } \\
\text { scattering } \\
\text { phenotype }\end{array}$ & $\begin{array}{c}\text { Induction } \\
\text { of IL-8 } \\
\text { secretion }\end{array}$ \\
\hline P1 wt & & + & + & + & + \\
\hline P12 wt & & + & + & + & + \\
\hline G27 wt & & + & + & + & + \\
\hline J99 wt & & + & - & - & + \\
\hline $\mathrm{P} 12 \Delta v a c A$ & HP0887 & + & + & + & + \\
\hline $\mathrm{P} 1 \Delta a \operatorname{lp} A / B$ & HР0912/HР0913 & + & - & - & - \\
\hline $\mathrm{P} 12 \Delta c a g$ PA1 & HP520-HP547 & - & - & - & - \\
\hline $\mathrm{P} 1 \Delta$ virB 4 & HP0544 & + & - & - & - \\
\hline $\mathrm{P} 1 \Delta v i r B 7$ & HP0532 & + & - & - & - \\
\hline $\mathrm{P} 1 \Delta v i r B 8$ & HР0529 & + & - & - & - \\
\hline $\mathrm{P} 1 \Delta$ virB 9 & HP0528 & + & - & - & - \\
\hline $\mathrm{P} 1 \Delta$ virB10 & HP0527 & + & - & - & - \\
\hline $\mathrm{P} 1 \Delta v i r B 11$ & HP0525 & + & - & - & - \\
\hline $\mathrm{P} 1 \Delta v i r D 4$ & HP0524 & + & - & - & + \\
\hline $\mathrm{P} 1 \Delta c a g A$ & HP0547 & - & - & - & + \\
\hline $\mathrm{G} 27 \Delta \mathrm{cag} F$ & HP0543 & + & - & - & - \\
\hline $\mathrm{P} 1 \Delta c a g H$ & HP0541 & + & - & - & - \\
\hline $\mathrm{G} 27 \Delta \operatorname{cag} I$ & HP0540 & + & - & - & - \\
\hline $\mathrm{G} 27 \Delta$ cagM & HP0537 & + & - & - & - \\
\hline $\mathrm{P} 1 \Delta \operatorname{cag} A / \operatorname{cag} A$ & HP0547 & + & + & + & + \\
\hline $\mathrm{P} 1 \Delta$ virD4/virD4 & HP0524 & + & + & + & + \\
\hline
\end{tabular}

${ }^{a}$ All H. pylori strains are type I isolates. cag PA1 mutagenesis and genetic complementation are described in Materials and Methods (see also Fig. 1). Bacterial infection was for $6 \mathrm{~h}$ at an MOI of 100. CagA translation and tyrosine phosphorylation were detected by immunoblot analysis. IL-8 production was determined by ELISA. wt, wild type

${ }^{b}$ From Tomb et al. (39).

tion and phosphorylation of CagA (Fig. 2B) and induction of the hummingbird phenotype (Fig. 3). This demonstrated that in our $\operatorname{cag} A$ and virD4 knockout mutants, the expression of flanking genes was not affected, and therefore, these mutations are not polar. Thus, both $c a g A$ and virD4 and possibly all other cag PAI genes investigated in this study played an essential role in translocation and phosphorylation of CagA and induction of the hummingbird phenotype, whereas $\operatorname{cag} A$ and $\operatorname{virD} 4$ were not essential for induction of IL-8 secretion.

\section{DISCUSSION}

Type IV secretion systems from several pathogenic bacteria have been shown to deliver bacterial factors into the cytosol of eukaryotic cells $(10,14)$. Virulent $H$. pylori strains carry a type IV secretion apparatus encoded in the cag PAI that has been shown to inject CagA and possibly also other virulence factors into the cytosol of infected host cells $(3,5,7,29,36,38)$. We have performed an extensive functional analysis of several $H$. pylori cag PAI genes and created isogenic knockout mutants. For the first time, two cag PAI genes ( $\operatorname{cag} A$ and virD4) were complemented on a shuttle vector. Our studies combined three different read-out systems and demonstrated the existence of a VirD4-CagA-independent host response (IL-8 secretion) and VirD4-CagA-dependent processes (translocation and tyrosine phosphorylation of CagA and induction of the scattering phenotype). These data considerably extend those of other groups $(1,9,23,40)$ and shed new light on the role and function of the H. pylori type IV secretion system during infection.
Our data showed that stimulation of IL-8 release, CagA translocation, and induction of phenotypic changes were dependent on many genes within the cag PAI. Specifically, all strains carrying knockout mutations in the $\operatorname{vir} B$ gene homologs were unable to elicit these host responses. This result strongly supports the view that $H$. pylori possesses a functional type IV secretion system that plays a crucial role in the host-pathogen interaction. As bacterial culture supernatants and bacteria defective in adherence (alp $A / B$ mutant) did not induce these effects, the type IV-mediated transport of $H$. pylori effector molecule(s) into the host cell cytoplasm is obviously a direct process that does not proceed through secretion into the extracellular medium.

Until now, the CagA protein has been the only known substrate for the $H$. pylori type IV secretion system. We observed that translocation of CagA was always correlated with cytoskeletal rearrangements, resulting in a scattering phenotype, suggesting that CagA triggers this host response (5; this study). The $\operatorname{cag} A$ and the virD4 gene knockout mutants did not induce changes in the actin cytoskeleton. However, knockout procedures by insertional mutagenesis can interfere with the expression of neighboring genes. Here we were able to exclude such polar effects by genetic complementation of our $\operatorname{cag} A$ and virD4 mutants, which indeed restored wild-type function.

VirD4 is a key component in the intensively studied type IV secretion system of the plant pathogen Agrobacterium tumefaciens. The agrobacterial VirD4 protein is located in the inner membrane (32) and is, in contrast to other Vir proteins, absolutely required for VirD2/T-DNA transfer into a wide range of host plants (24). VirD4 contains a Walker A nucleotide triphosphate binding motif that is necessary for function. Consequently, VirD4 is thought to mediate introduction of the nucleoprotein complex into the transporter by an energy-dependent mechanism (44). As the $H$. pylori virD4 gene shows significant homology to its counterpart in A. tumefaciens, the function of these proteins is probably similar. Thus, the $H$. pylori VirD4 protein presumably acts as an adapter protein for the transfer of CagA from the bacterial cell into the transfer channel formed by proteins encoded by the other vir genes.

Apart from inducing host cytoskeletal rearrangements, the $H$. pylori type IV secretion system has been speculated to play a role in the induction of proinflammatory host responses. However, until now there has been no systematic approach analyzing all vir genes. We used four well-characterized $H$. pylori strains and demonstrated that virB4, virB7, virB8, virB9, virB10, virB11, cagI, cagF, $c a g H$, and $c a g M$ are involved in proinflammatory responses, suggesting that the integrity of the type IV secretion system is essential. Yet the only bacterial factor known to be translocated through the type IV machinery, CagA, does not induce proinflammatory signaling, as the $\operatorname{cag} A$ knockout mutant induced wildtype levels of IL- 8 secretion. These observations strongly suggest that $H$. pylori induces IL- 8 secretion by the transfer of another, as yet unknown effector molecule.

In this context it is interesting that our virD4 gene knockout mutant induced intermediate levels of IL- 8 secretion. This observation contrasts with the finding that a virD4 knockout mutant of the TIGR strain 26695 shows 3.6-fold increased IL-8 secretion (16). The reason for this discrepancy is not clear. The possibility that our mutant is polar, however, can be ruled out. Perhaps the different findings are the result of differences 
Wild-type

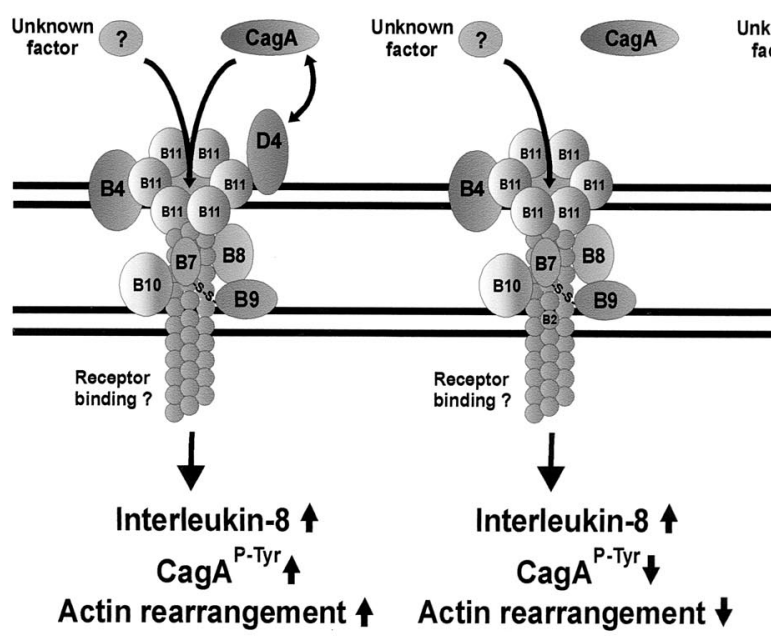

CagA mutant
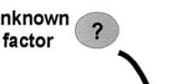
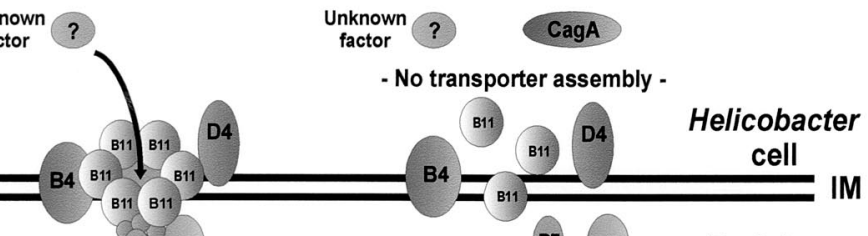

B11 B11

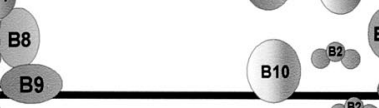

B7 B8 Periplasm

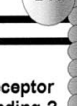

inding ?
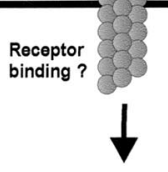

Interleukin-8 †

$\mathrm{CagA}^{\mathrm{P}-\mathrm{Tyr}} \downarrow$

Actin rearrangement $\downarrow$ Actin rearrangement $\downarrow$

FIG. 4. Hypothetical model for the induction of type IV-dependent host responses by H. pylori. The type IV secretion system consists of VirB proteins (B4 and B7 to B11), which assemble in the inner (IM) and outer (OM) bacterial membranes. VirD4 is thought to act as an adapter protein, guiding CagA into the transport channel. Translocated CagA is tyrosine phosphorylated $\left(\mathrm{Cag} \mathrm{A}^{\mathrm{P}-\mathrm{Tyr}} \uparrow\right)$ and induces actin cytoskeletal rearrangements associated with the scattering phenotype. cagA and virD4 mutants are unable to translocate CagA, and hence, rearrangement of the host cell cytoskeleton failed. Stimulation of IL-8 secretion, however, is VirD4-CagA independent. It is induced either by the translocation of an as yet unidentified factor or by binding of the transporter itself to relevant cell surface receptors (receptor hypothesis). virB mutants do not assemble a functional transporter and therefore are unable to elicit any of these host responses. For more details, see the text.

between the two wild-type strains used in the study of Crabtree and coworkers and our study (26695 and P1, respectively). Those authors suggested that VirD4 is a bacterial downregulator (modulator) of IL-8 synthesis. An important common result from both studies, however, is that VirD4 plays a role in $H$. pylori-induced host responses that is different from those of the other vir genes. In particular, inactivation of virD4 prevented CagA translocation and phosphorylation but did not block proinflammatory responses.

Collectively, our results suggest that both CagA translocation and induction of IL-8 release are dependent on the type IV secretion system. However, the mechanism is apparently not identical, as the role of virD4 in these processes is different. If IL- 8 secretion is induced by another translocated bacterial factor, then this unidentified factor does not depend on VirD4 in order to be transferred to the type IV secretion channel. Together with preliminary biochemical evidence (22; unpublished data), these observations suggest that the factor may not even be a protein. Alternatively, Covacci and Rappuoli suggested that proinflammatory responses could be induced by the type IV secretion channel itself, which could perturb membranes (13), or bind to and activate a relevant cell surface receptor(s), known as the receptor hypothesis.

The model presented in Fig. 4 summarizes the results obtained in this study. The $H$. pylori type IV secretion system spans both bacterial membranes. This needle-like structure is thought to inject effector molecules into the cytoplasm of host cells. However, a typical pilus protein is unknown, as a virB2 homologue is absent from the $H$. pylori genome $(2,39)$. We suggest that VirD4 is located at the cytoplasmic face of the inner membrane (similar to its agrobacterial counterpart) and guides CagA to the translocation channel.

We have shown here that the type IV secretion system medi- ates both VirD4-CagA-dependent and VirD4-CagA-independent host responses. CagA phosphorylation and rearrangements of the host actin cytoskeleton depended on CagA translocation. Thus, virD4 and $\operatorname{cag} A$ mutants are unable to elicit these host responses (Fig. 4). IL-8 secretion is a VirD4-CagA-independent process and is induced by either (i) translocation of an unknown factor or (ii) direct binding of the type IV channel to the host membrane or a cellular receptor. Currently it is unclear which of the two possibilities holds true. However, recent studies with animal models and clinical isolates have shown the existence of strains inducing IL-8 secretion regardless of the cag PAI structure $(4,18)$. This argues against a mechanism of IL-8 secretion induced by the type IV system directly.

We favor a model in which $H$. pylori translocates an unknown effector molecule into the cytoplasm of host cells. Yet the transfer mechanism is substantially different from CagA transfer and may under some circumstances even be cag PAI independent. Purification and identification of this unknown factor will shed new light on the pathogenic mechanisms of this important human pathogen.

\section{ACKNOWLEDGMENTS}

We are grateful to Antonello Covacci and Stefano Censini (ISIC, Siena, Italy) and Rainer Haas (Max-von-Pettekofer-Institut, Munich, Germany) for providing us with anti-CagA antibody and $H$. pylori mutant strains. We thank Katharina Krüger for generation of the $c a g H$ mutant.

This work was supported by a grant from the Fonds der Chemischen Industrie to T.F.M.

\section{REFERENCES}

1. Akopyants, N. S., S. W. Clifton, D. Kersulyte, J. E. Crabtree, B. E. Youree, C. A. Reece, N. O. Bukanov, E. S. Drazek, B. A. Roe, and D. E. Berg. 1998. Analyses of the cag pathogenicity island of Helicobacter pylori. Mol. Microbiol. 28:37-53. 
2. Alm, R. A., L. S. Ling, D. T. Moir, B. L. King, E. D. Brown, P. C. Doig, D. R. Smith, B. Noonan, B. C. Guild, B. L. deJonge, G. Carmel, P. J. Tummino, A. Caruso, M. Uria-Nickelsen, D. M. Mills, C. Ives, R. Gibson, D. Merberg, S. D. Mills, Q. Jiang, D. E. Taylor, G. F. Vovis, and T. J. Trust. 1999 Genomic-sequence comparison of two unrelated isolates of the human gastric pathogen Helicobacter pylori. Nature 397:176-180.

3. Asahi, M., T. Azuma, S. Ito, Y. Ito, H. Suto, Y. Nagai, M. Tsubokawa, Y. Tohyama, S. Maeda, M. Omata, T. Suzuki, and C. Sasakawa. 2000. Helicobacter pylori CagA protein can be tyrosine phosphorylated in gastric epithelial cells. J. Exp. Med. 191:593-602.

4. Audibert, C., C. Burucoa, B. Janvier, and J. L. Fauchere. 2001. Implication of the structure of the Helicobacter pylori cag pathogenicity island in induction of interleukin-8 secretion. Infect. Immun. 69:1625-1629.

5. Backert, S., S. Moese, M. Selbach, V. Brinkmann, and T. F. Meyer. 2001 Phosphorylation of tyrosine 972 of the Helicobacter pylori CagA protein is essential for induction of a scattering phenotype in gastric epithelial cells. Mol. Microbiol. 42:631-644.

6. Backert, S., E. C. Müller, P. R. Jungblut, and T. F. Meyer. 2001. Tyrosine phosphorylation patterns and size modification of the Helicobacter pylori CagA protein after translocation into gastric epithelial cells. Proteomics 1:608-617.

7. Backert, S., E. Ziska, V. Brinkmann, U. Zimny-Arndt, A. Fauconnier, P. R Jungblut, M. Naumann, and T. F. Meyer. 2000. Translocation of the Helicobacter pylori CagA protein in gastric epithelial cells by a type IV secretion apparatus. Cell. Microbiol. 2:155-164.

8. Bodger, K., and J. E. Crabtree. 1998. Helicobacter pylori and gastric inflammation. Br. Med. Bull. 54:139-150.

9. Censini, S., C. Lange, Z. Xiang, J. E. Crabtree, P. Ghiara, M. Borodovsky, R. Rappuoli, and A. Covacci. 1996. Cag, a pathogenicity island of Helicobacter pylori, encodes type I-specific and disease-associated virulence factors. Proc. Natl. Acad. Sci. USA 93:14648-14653.

10. Christie, P. J., and J. P. Vogel. 2000. Bacterial type IV secretion: conjugation systems adapted to deliver effector molecules to host cells. Trends Microbiol. 8:354-360.

11. Churin, Y., E. Kardalinou, T. F. Meyer, and M. Naumann. 2001. Pathogenicity island-dependent activation of Rho GTPases Rac1 and Cdc42 in $\mathrm{Hel}$ icobacter pylori infection. Mol. Microbiol. 40:815-823.

12. Covacci, A., S. Censini, M. Bugnoli, R. Petracca, D. Burroni, G. Macchia, A. Massone, E. Papini, Z. Xiang, and N. Figura. 1993. Molecular characterization of the $128-\mathrm{kDa}$ immunodominant antigen of Helicobacter pylori associated with cytotoxicity and duodenal ulcer. Proc. Natl. Acad. Sci. USA 90: 5791-5795.

13. Covacci, A., and R. Rappuoli. 2000. Tyrosine-phosphorylated bacterial proteins: Trojan horses for the host cell. J. Exp. Med. 191:587-592.

14. Covacci, A., J. L. Telford, G. Del Giudice, J. Parsonnet, and R. Rappuoli. 1999. Helicobacter pylori virulence and genetic geography. Science $\mathbf{2 8 4}$ :13281333.

15. Cover, T. L., and M. J. Blaser. 1999. Helicobacter pylori factors associated with disease. Gastroenterology 117:257-261.

16. Crabtree, J. E., D. Kersulyte, S. D. Li, I. J. Lindley, and D. E. Berg. 1999. Modulation of Helicobacter pylori induced interleukin-8 synthesis in gastric epithelial cells mediated by cag PAI encoded VirD4 homologue. J. Clin. Pathol. 52:653-657.

17. de Bernard, M., M. Moschioni, G. Napolitani, R. Rappuoli, and C. Montecucco. 2000. The VacA toxin of Helicobacter pylori identifies a new intermediate filament-interacting protein. EMBO J. 19:48-56.

18. Eaton, K. A., D. Kersulyte, M. Mefford, S. J. Danon, S. Krakowka, and D. E. Berg. 2001. Role of Helicobacter pylori cag region genes in colonization and gastritis in two animal models. Infect. Immun. 69:2902-2908.

19. Foryst-Ludwig, A., and M. Naumann. 2000. p21-activated kinase 1 activates the nuclear factor kappa B (NF-кB)-inducing kinase-Ikappa B kinases $\mathrm{NF}-\kappa \mathrm{B}$ pathway and proinflammatory cytokines in Helicobacter pylori infection. J. Biol. Chem. 275:39779-39785.

20. Heuermann, D., and R. Haas. 1998. A stable shuttle vector system for efficient genetic complementation of Helicobacter pylori strains by transformation and conjugation. Mol. Gen. Genet. 257:519-528.

21. Ilver, D., A. Arnqvist, J. Ogren, I. M. Frick, D. Kersulyte, E. T. Incecik, D. E. Berg, A. Covacci, L. Engstrand, and T. Boren. 1998. Helicobacter pylori adhesin binding fucosylated histo-blood group antigens revealed by retagging. Science 279:373-377.

22. Kassai, K., T. Yoshikawa, N. Yoshida, A. Hashiramoto, M. Kondo, and H. Murase. 1999. Helicobacter pylori water extract induces interleukin-8 production by gastric epithelial cells. Dig. Dis. Sci. 44:237-242.

23. Li, S. D., D. Kersulyte, I. J. Lindley, B. Neelam, D. E. Berg, and J. E. Crabtree. 1999. Multiple genes in the left half of the cag pathogenicity island of Helicobacter pylori are required for tyrosine kinase-dependent transcription of interleukin-8 in gastric epithelial cells. Infect. Immun. 67:3893-3899.
24. Lin, T. S., and C. I. Kado. 1993. The virD4 gene is required for virulence while virD3 and orf5 are not required for virulence of Agrobacterium tumefaciens. Mol. Microbiol. 9:803-812.

25. Lindholm, C., M. Quiding-Jarbrink, H. Lonroth, A. Hamlet, and A. M. Svennerholm. 1998. Local cytokine response in Helicobacter pylori-infected subjects. Infect. Immun. 66:5964-5971.

26. Moese, S., M. Selbach, U. Zimny-Arndt, P. R. Jungblut, T. F. Meyer, and S. Backert. 2001. Identification of a tyrosine-phosphorylated $35 \mathrm{kDa}$ carboxyterminal fragment $\left(\mathrm{p} 35^{\mathrm{CagA}}\right)$ of the Helicobacter pylori CagA protein in phagocytic cells: processing or breakage? Proteomics 1:618-629.

27. Montecucco, C., and R. Rappuoli. 2000. Living dangerously: how Helicobacter pylori survives in the human stomach. Nat. Rev. Mol. Cell. Biol. 2:457466.

28. Odenbreit, S., B. Gebert, J. Püls, W. Fischer, and R. Haas. 2001. Interaction of Helicobacter pylori with professional phagocytes: role of the cag pathogenicity island and translocation, phosphorylation and processing of CagA. Cell. Microbiol. 3:21-32.

29. Odenbreit, S., J. Püls, B. Sedlmaier, E. Gerland, W. Fischer, and R. Haas. 2000. Translocation of Helicobacter pylori CagA into gastric epithelial cells by type IV secretion. Science 287:1497-1500.

30. Odenbreit, S., M. Till, D. Hofreuter, G. Faller, and R. Haas. 1999. Genetic and functional characterization of the $\operatorname{alp} A B$ gene locus essential for the adhesion of Helicobacter pylori to human gastric tissue. Mol. Microbiol. 31:1537-1548.

31. Ogura, K., S. Maeda, M. Nakao, T. Watanabe, M. Tada, T. Kyutoku, H. Yoshida, Y. Shiratori, and M. Omata. 2000. Virulence factors of Helicobacter pylori responsible for gastric diseases in Mongolian gerbil. J. Exp. Med. 192:1601-1610.

32. Okamoto, S., A. Toyoda-Yamamoto, K. Ito, I. Takebe, and Y. Machida. 1991. Localization and orientation of the VirD4 protein of Agrobacterium tumefaciens in the cell membrane. Mol. Gen. Genet. 228:24-32.

33. Reyrat, J. M., V. Pelicic, E. Papini, C. Montecucco, R. Rappuoli, and J. L. Telford. 1999. Towards deciphering the Helicobacter pylori cytotoxin. Mol. Microbiol. 34:197-204

34. Rieder, G., R. A. Hatz, A. P. Moran, A. Walz, M. Stolte, and G. Enders. 1997. Role of adherence in interleukin-8 induction in Helicobacter pylori-associated gastritis. Infect. Immun. 65:3622-3630.

35. Schmitt, W., and R. Haas. 1994. Genetic analysis of the Helicobacter pylori vacuolating cytotoxin: structural similarities with the IgA protease type of exported protein. Mol. Microbiol. 12:307-319.

36. Segal, E. D., J. Cha, J. Lo, S. Falkow, and L. S. Tompkins. 1999. Altered states: involvement of phosphorylated CagA in the induction of host cellular growth changes by Helicobacter pylori. Proc. Natl. Acad. Sci. USA 96:1455914564.

37. Sharma, S. A., M. K. Tummuru, M. J. Blaser, and L. D. Kerr. 1998. Activation of IL-8 gene expression by Helicobacter pylori is regulated by transcription factor nuclear factor- $\kappa \mathrm{B}$ in gastric epithelial cells. J. Immunol. 160:2401-2407.

38. Stein, M., R. Rappuoli, and A. Covacci. 2000. Tyrosine phosphorylation of the Helicobacter pylori CagA antigen after cag-driven host cell translocation. Proc. Natl. Acad. Sci. USA 97:1263-1268.

39. Tomb, J. F., O. White, A. R. Kerlavage, R. A. Clayton, G. G. Sutton, R. D. Fleischmann, K. A. Ketchum, H. P. Klenk, S. Gill, B. A. Dougherty, K. Nelson, J. Quackenbush, L. Zhou, E. F. Kirkness, S. Peterson, B. Loftus, D. Richardson, R. Dodson, H. G. Khalak, A. Glodek, K. McKenney, L. M. Fitzegerald, N. Lee, M. D. Adams, E. K. Hickey, D. E. Berg, J. D. Gocayne, T. R. Utterback, J. D. Peterson, J. M. Kelley, M. D. Cotton, J. M. Weidman, C. Fujii, C. Bowman, L. Watthey, E. Wallin, W. S. Hayes, M. Borodowsky, P. D. Karp, H. O. Smith, C. M. Fraser, and J. C. Venter. 1997. The complete genome sequence of the gastric pathogen Helicobacter pylori. Nature 388: 539-547.

40. Tummuru, M. K., S. A. Sharma, and M. J. Blaser. 1995. Helicobacter pylori picB, a homologue of the Bordetella pertussis toxin secretion protein, is required for induction of IL-8 in gastric epithelial cells. Mol. Microbiol. 18:867-876.

41. Wilson, M., R. Seymour, and B. Henderson. 1998. Bacterial perturbation of cytokine networks. Infect. Immun. 66:2401-2409.

42. Yamaoka, Y., M. Kita, T. Kodama, N. Sawai, T. Tanahashi, K. Kashima, and J. Imanishi. 1998. Chemokines in the gastric mucosa in Helicobacter pylori infection. Gut 42:609-617.

43. Yeo, H. J., S. N. Savvides, A. B. Herr, E. Lanka, and G. Waksman. 2000 Crystal structure of the hexameric traffic ATPase of the Helicobacter pylori type IV secretion system. Mol. Cell 6:1461-1472.

44. Zupan, J. R., D. Ward, and P. Zambryski. 1998. Assembly of the VirB transport complex for DNA transfer from Agrobacterium tumefaciens to plant cells. Curr. Opin. Microbiol. 1:649-655. 